\title{
Linear magnetoresistance in compensated graphene bilayer
}

\author{
G. Yu. Vasileva, ${ }^{1,2,3}$ D. Smirnov, ${ }^{1}$ Yu. L. Ivanov, ${ }^{2}$ Yu. B. Vasilyev, ${ }^{2}$ P. S. Alekseev, ${ }^{2}$ A. P. Dmitriev, ${ }^{2}$ I. V. Gornyi, ${ }^{2,4,5}$ \\ V. Yu. Kachorovskii, ${ }^{2}$ M. Titov, ${ }^{6}$ B. N. Narozhny, ${ }^{5,7}$ and R. J. Haug ${ }^{1}$ \\ ${ }^{1}$ Institut für Festkörperphysik, Leibniz Universität Hannover, Appelstraße 2, D-30167 Hannover, Germany \\ ${ }^{2}$ Ioffe Institute, Polytechnicheskaya 26, 194021 St. Petersburg, Russia \\ ${ }^{3}$ Peter the Great Saint-Petersburg Polytechnic University, Politechnicheskaya 29, 195251 St. Petersburg, Russia \\ ${ }^{4}$ Institut für Nanotechnologie, Karlsruhe Institute of Technology, D-76021 Karlsruhe, Germany \\ ${ }^{5}$ Institut für Theorie der Kondensierten Materie, Karlsruhe Institute of Technology, D-76128 Karlsruhe, Germany \\ ${ }^{6}$ Institute for Molecules and Materials, Radboud University Nijmegen, NL-6525 AJ Nijmegen, The Netherlands \\ ${ }^{7}$ National Research Nuclear University MEPhI (Moscow Engineering Physics Institute), 115409 Moscow, Russia
}

(Received 2 December 2015; revised manuscript received 22 April 2016; published 20 May 2016)

\begin{abstract}
We report a nonsaturating linear magnetoresistance in charge-compensated bilayer graphene in a temperature range from 1.5 to $150 \mathrm{~K}$. The observed linear magnetoresistance disappears away from charge neutrality, ruling out the traditional explanation of the effect in terms of the classical random resistor network model. We show that experimental results qualitatively agree with a phenomenological two-fluid model taking into account electron-hole recombination and finite-size sample geometry.
\end{abstract}

DOI: 10.1103/PhysRevB.93.195430

\section{INTRODUCTION}

Classical magnetoresistance is a perfect tool for experimental studies of multicomponent electronic systems [1] where the conventional theory of electronic transport [2] predicts a quadratic dependence of the resistance on the weak applied magnetic field followed by a saturation in classically strong fields. While most materials do exhibit quadratic behavior [3], there is a fast growing number of experiments reporting observations of linear magnetoresistance (LMR) in a wide variety of novel materials, including multilayer graphenes [4-6], topological insulators [7-14], Dirac [15-19] and Weyl [20,21] semimetals, transition-metal dichalcogenides [22], as well as in narrow-gap semiconductors [23] and three-dimensional (3D) silver chalcogenides [24,25].

Semiclassical linear magnetoresistance has been predicted for 3D metallic slabs with complex Fermi surfaces and smooth boundaries [26,27], for strongly inhomogeneous, granular materials [28], and for compensated two-component systems with quasiparticle recombination [29]. Purely quantum effects (and screening of charged impurities) lead to LMR in zero-gap band systems with linear dispersion in the case where all carriers belong to the first Landau level [30-32]. In weak fields, quantum interference in two-dimensional electron systems yields an interaction correction [33] to resistivity that is linear in the Zeeman magnetic field.

The extreme quantum limit of Refs. [30,31] has been realized in graphene [6] and in $\mathrm{Bi}_{2} \mathrm{Se}_{3}$ nanosheets [12]. The quantum theory was also reported [7] to be applicable to the novel topological material LuPdBi. The classical theory of Ref. [28] was recently used to interpret the behavior of hydrogen-intercalated epitaxial bilayer graphene [4]. It was argued that large samples of epitaxial bilayer graphene contain a "built-in mosaic tiling" due to dense dislocation networks [34], making it an ideal material to realize the random network model of Ref. [28]. At the same time, neither theory can explain LMR in homogeneous topological insulators [35] and neutral two-component systems $[8,11,23]$.
In this paper we report the results of a systematic experimental analysis of magnetotransport in exfoliated bilayer graphene. Precisely at charge neutrality, we have observed nonsaturating LMR in a wide range of magnetic fields in Hall bars of widths $0.5,0.95$, and $2.0 \mu \mathrm{m}$ in a temperature range from 1.5 to $150 \mathrm{~K}$. Deviations from charge neutrality lead to eventual saturation of the magnetoresistance. Our key experimental findings are not accounted for within the random resistor network model [28]. Indeed, this model is insensitive to the relative concentration of different types of charge carriers and thus cannot explain the observed saturation of the magnetoresistance away from charge neutrality. This model also does not explain the transition between the quadratic dependence at very weak magnetic fields and LMR observed at higher fields [8]. The extreme quantum limit is unlikely to be reached in our system at $150 \mathrm{~K}$ for both electrons and holes [8,35]. Moreover, the excitation spectrum in bilayer graphene is quadratic, which rules out the quantum theory of Refs. [30,31].

We are able to explain our results in terms of a semiclassical description of finite-size, charge-compensated two-component systems in moderately strong, classical magnetic fields [8,29]. The key element of the physical picture of Ref. [29] is the electron-hole recombination [36]. When an external magnetic field is applied, recombination processes allow for a neutral quasiparticle flow in the lateral direction relative to the electric current [37]. Although such neutral current cannot be directly detected in our measurements, its presence leads to a redistribution of charge carriers over the sample area, influencing the nonuniform profile of the electric current in the sample. As a result, the sample is essentially split into the bulk and edge regions, which contribute to the total sheet resistance of the sample as parallel resistors. The bulk and edge resistances exhibit qualitatively different dependencies on the magnetic field, yielding LMR. Away from charge neutrality, a nonzero Hall voltage is formed, leading to the observed saturation of the magnetoresistance. 


\section{EXPERIMENTAL DETAILS AND SAMPLE CHARACTERIZATION}

We have prepared the sample by placing the exfoliated bilayer graphene sheet on a substrate consisting of a highly doped $\mathrm{Si}$ wafer covered by a 330-nm-thick $\mathrm{SiO}_{2}$ film. Subsequently, the sample was patterned into a triple Hall bar device, as seen in an atomic force microscope (AFM) image [Fig. 1(b)]. The sample consists of three sections $2,0.95$, and $0.5 \mu \mathrm{m}$ wide. The length of each Hall bar is $1.8 \mu \mathrm{m}$. The sample was purified using an AFM tip (instead of annealing) which allows one to considerably decrease the concentration of charged impurities on top of the graphene. The carrier concentration $n$ in the sample can be varied up to $5 \times 10^{12} \mathrm{~cm}^{-2}$ by applying a gate voltage $V_{g}$ to the conducting substrate, which acts as a back gate.

Magnetotransport was studied by a four-probe method with simultaneous measurements of longitudinal $R_{x x}$ and transverse $R_{x y}$ resistances in perpendicular magnetic fields from 0 to $7 \mathrm{~T}$ and in a temperature range from 1.5 to $150 \mathrm{~K}$ passing an ac current with an amplitude of $10 \mu \mathrm{A}$ through the sample.

To characterize the sample and to define the charge neutrality point (CNP), the field effect (FE) was measured for each section of the device. Figure 1(a) shows the FE dependences measured at $B=0 \mathrm{~T}$ and $T=25 \mathrm{~K}$ for the three sections of the device. All three sections exhibit a graphene typical FE with a sharp maximum corresponding to the CNP. The precise value of $V_{g}^{*}$ corresponding to the CNP depends on the Hall bar width and is shifted from $0.8 \mathrm{~V}$ in the widest section of the sample toward $3.6 \mathrm{~V}$ in the medium and $10.4 \mathrm{~V}$ in the narrowest Hall bar. The maximum resistivity in the widest and middle sections is $5.6 \mathrm{k} \Omega$ while it exceeds $6.2 \mathrm{k} \Omega$ for the narrowest section.

Far away from the CNP only one band contributes to electronic transport and hence the carrier mobility can be estimated with the help of the usual one-band model. The estimate requires the knowledge of the charge density shown in Fig. 1(c) for $T=1.5 \mathrm{~K}$ (far away from the CNP the charge density coincides with the carrier density). We have measured the charge density by three independent methods [i.e., using
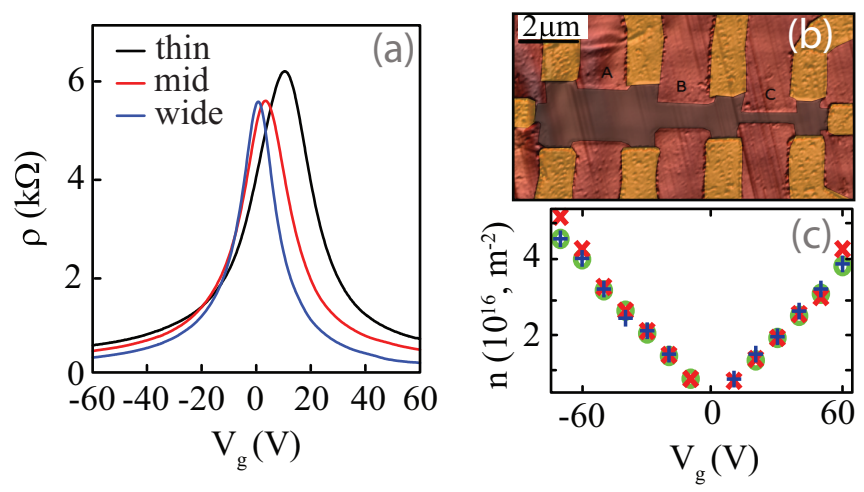

FIG. 1. (a) The gate voltage dependence of the resistivity measured at $B=0 \mathrm{~T}$ and $T=25 \mathrm{~K}$ for the three sections of the device. (b) The AFM image of the sample (gray) with contacts (yellow). The sample contains three Hall bar sections $2,0.95$, and $0.5 \mu \mathrm{m}$ wide (left to right). (c) Charge density extracted from the period of the SdH oscillations (blue “+”), from the Hall effect (red " $\times$ ”) and from the capacity model (green " $\bigcirc$ ").
Shubnikov-de Haas $(\mathrm{SdH})$ oscillations, the classical Hall effect, and the capacity model], yielding almost identical results. The resulting mobilities increase with the width of the sample; we have obtained the following values for the mobilities of the narrowest, medium, and widest sections: 2200,3000 , and $4000 \mathrm{~cm}^{2} / \mathrm{V}$ s for holes and 2600,3000 , and $3800 \mathrm{~cm}^{2} / \mathrm{V}$ s for electrons, respectively.

In proximity to the CNP, the one-band model fails and one needs to consider the two-band model of electronic transport. In the disorder-dominated regime, the two-band conductivity is given by the sum of two independent contributions due to electrons and holes. Assuming fast enough relaxation, we can describe both bands by the same chemical potential. To leading order, the chemical potential is proportional to the gate voltage. The electron and hole mobilities can now be found by fitting the resistance curves shown in Fig. 1(a). This procedure yields the mobilities which are very close to the above values. Hence, the electron and hole mobilities are practically independent of the gate voltage.

The dependence of the mobility on the width of the sample can be attributed to the fact that near the sample edges the mobility is significantly reduced due to scattering off the edges themselves as well as the additional defects inherent to the boundary region $[38,39]$. Although the above mobilities are not very high, the samples are of a good quality, having a clear manifestation of CNP and exhibiting the quantum Hall effect. Measurements of the Hall resistance in the wide section of the sample at $1.5 \mathrm{~K}$ in a magnetic field of $9.5 \mathrm{~T}$ demonstrate the features inherent to bilayer graphene following from filling factors in the Hall plateaus equal to $v= \pm 4, \pm 8, \pm 12$. In a strong magnetic field the neutrality point is shifted towards higher gate voltages (see Fig. 2). For the wide section of the sample at $12 \mathrm{~T}$, the CNP corresponds to $V_{g}=7 \mathrm{~V}$. This effect has also been observed in other sections of the sample.

\section{LINEAR MAGNETORESISTANCE}

We have measured the longitudinal resistance for all three sections of the sample and the Hall resistance between the widest and medium sections in the interval of gate voltages from -20 to $32.2 \mathrm{~V}$ with a step $\delta V_{g}=2.4 \mathrm{~V}$ that includes

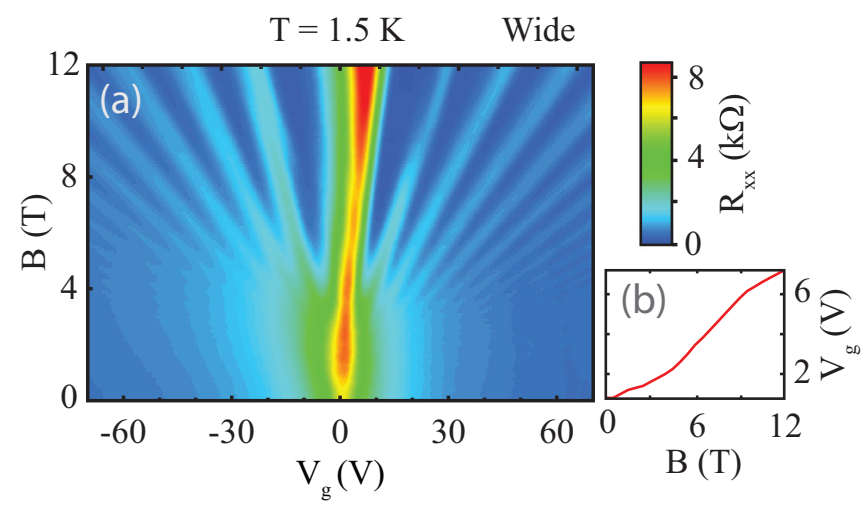

FIG. 2. Color plot of $R_{x x}$ in the wide sample at $T=1.5 \mathrm{~K}$ as a function of magnetic field and gate voltage (a). The fanlike peak structure clearly demonstrates the Landau levels. The central peak shows the shift of the charge neutrality point with magnetic field (b). 
the CNP for all three sections. The data for the wide section of the sample at $T=1.5 \mathrm{~K}$ are shown in Fig. 2. To reduce the conductance fluctuations, further measurements were performed at higher temperatures: 25, 50, 100, and $150 \mathrm{~K}$. At such high temperatures the quantum effects, e.g., Landau quantization, are not detectable in our samples while the applied magnetic fields are not too strong, $B<7 \mathrm{~T}$.

The magnetoresistance data for the thin section of the sample at the four temperatures are shown in Fig. 3. The data show linear behavior close to the neutrality point (the black curve corresponding to the gate voltage $V_{g}=10.6 \mathrm{~V}$ ). Away from neutrality, the data show linear behavior for an intermediate range of magnetic fields, followed by a saturation at stronger fields. Similar results were obtained for the other two sections of the sample. At the same time, the Hall resistance grows in amplitude in strong fields (see Fig. 4).

Although these observations are in good qualitative agreement with the theoretical predictions of Ref. [29], there are several additional factors that may (and probably do) conspire to yield the observed behavior. The charge neutrality point in our samples shifts towards higher gate voltages in a high magnetic field. As a result, the same value of $V_{g}$ may correspond to different carrier densities in low and high fields. Shifting away from charge neutrality can cause both the saturation of $R_{x x}(B)$, as exhibited by most of the curves in Fig. 3, and the nonzero Hall resistivity [see Fig. 4(b)]. The latter can also be due to electron-hole asymmetry in the sample, where the mobilities of electrons and holes are sufficiently different $[40,41]$. Both effects may appear if the sample contained macroscopic inhomogeneities or resonant impurities, which strongly modify the density of states near charge neutrality. Finally, in contrast to the theory worked out in Ref. [29], the length of our samples is comparable to their width and the samples cannot be considered infinitely long.
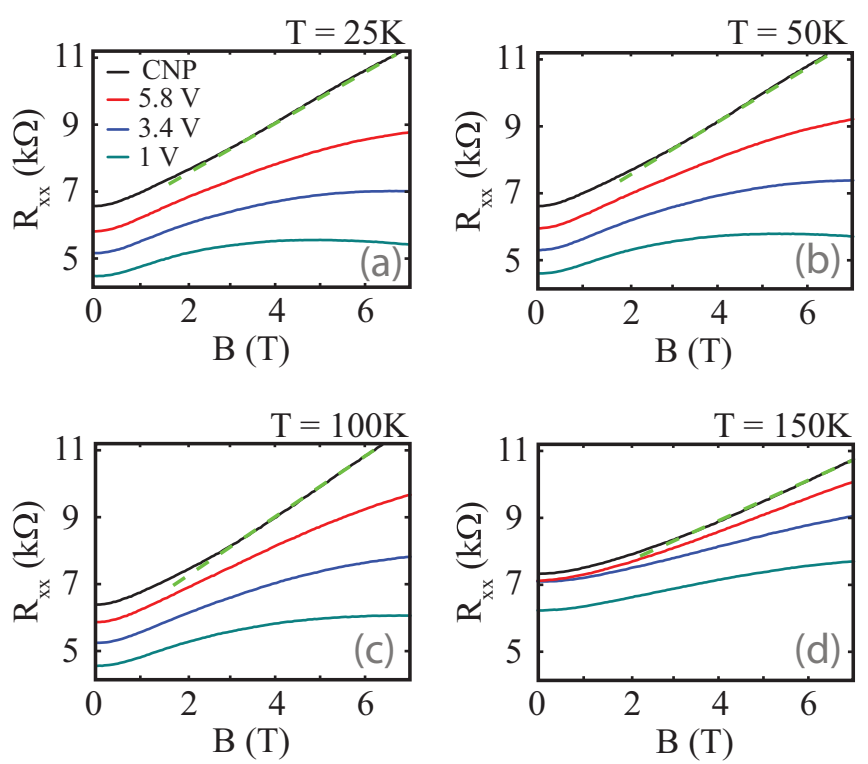

FIG. 3. Magnetoresistance of the thin section of the sample at (a) 25, (b) 50, (c) 100, and (d) $150 \mathrm{~K}$ for several gate voltages indicated on the plot. The green dashed lines are linear guides to the eyes.
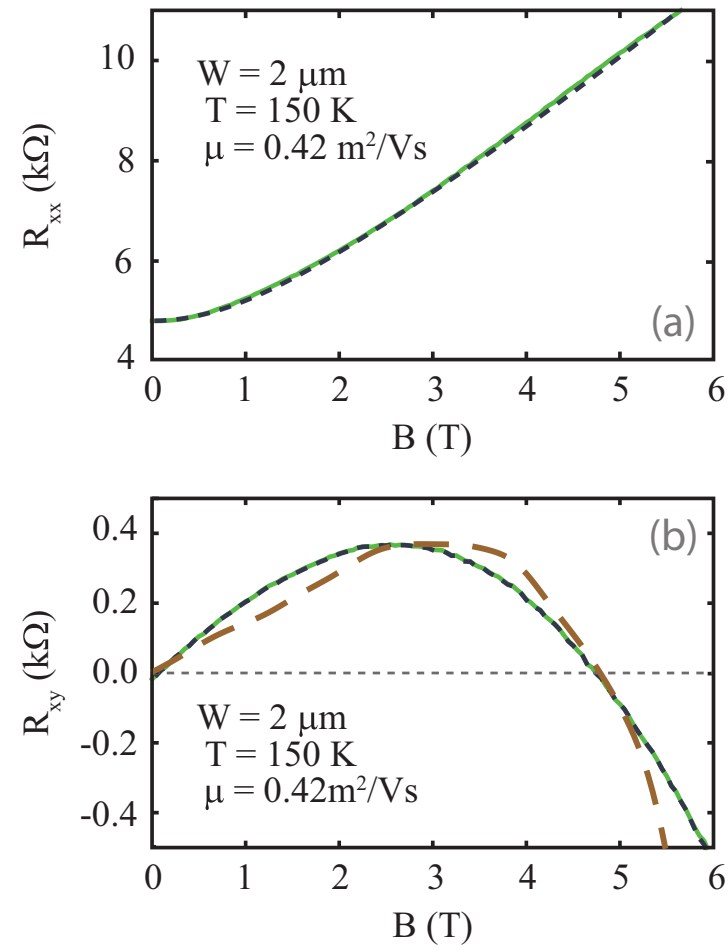

FIG. 4. (a) Magnetoresistance of the wide section of the sample at $T=150 \mathrm{~K}$ and $V_{g}=1 \mathrm{~V}$, closest to the charge neutrality point. The solid (green) line represents the experimental data; the dashed (blue) line represents the theoretical fit using the semiclassical description adapted from Ref. [29] [see Eqs. (1)], with the parameters given in Table I. (b) Hall resistance of the wide section of the sample at $T=150 \mathrm{~K}$ and $V_{g}=1 \mathrm{~V}$. The solid (green) line represents the experimental data; the dashed (blue) line represents a consistency check for the theory (1) where the carrier density was obtained from the experimental values of $R_{x y} / R_{x x}(B)$; the brown curve shows the theoretical fit where the carrier density was recalculated from the observed dependence of the maximum resistance (i.e., CNP) on the magnetic field (see Figs. 1 and 2).

Some of the above complications present a significant challenge for an analytic theory. Nevertheless, we may attempt to analyze the measured data with the help of the existing theory of Ref. [29]. The simplest version of this theory (applicable to a particle-hole symmetric system with parabolic dispersion and energy-independent impurity scattering rate) yields the following expressions for the longitudinal and Hall resistivities of a two-component system near charge neutrality:

$$
\begin{aligned}
& R_{x x}=R_{0} \frac{1+\mu^{2} B^{2}}{1+\mu^{2} B^{2}\left[\frac{\tanh \left(W / \ell_{R}\right)}{W / \ell_{R}}\left(1-\frac{n^{2}}{\rho^{2}}\right)+\frac{n^{2}}{\rho^{2}}\right]}, \\
& R_{x y}=\frac{R_{0} n}{\rho} \frac{\left(1+\mu^{2} B^{2}\right) \mu B}{1+\mu^{2} B^{2}\left[\frac{\tanh \left(W / \ell_{R}\right)}{W / \ell_{R}}\left(1-\frac{n^{2}}{\rho^{2}}\right)+\frac{n^{2}}{\rho^{2}}\right]} .
\end{aligned}
$$

Here, $n$ and $\rho$ are the charge and quasiparticle densities, $\mu$ is the mobility (which is assumed to be the same for both electrons and holes), $W$ is the sample width, $R_{0}$ is the zerofield resistivity, and $\ell_{R}$ is the field-dependent recombination length. Assuming that the dominant recombination process is the impurity-assisted electron-phonon coupling that can 
occur anywhere in the sample with equal probability, the recombination length found in Ref. [29] is given by

$$
\ell_{R}=\frac{\ell_{0}}{\sqrt{1+\mu^{2} B^{2}}}, \quad \ell_{0}=2 \sqrt{D \tau_{R}},
$$

where $D$ is the diffusion coefficient and $\tau_{R}$ is the recombination time in zero magnetic field. As a result, in classically strong fields $(\mu B \gg 1)$ and for $W \gg \ell_{R}$, the magnetoresistance (1a) close to the charge neutrality point is linear, $R_{x x} \approx R_{0} W \mu B / \ell_{0}$.

The theory of Ref. [29] yields a quadratic magnetoresistance in the weak field and a linear dependence for the classically strong magnetic field, in qualitative agreement with our data. However, this simple theory does not quantitatively describe our results in the whole range of magnetic fields used in our experiments. At the same time, our data can be quantitatively described by Eqs. (1a) and (1b) if we introduce an empiric expression for the recombination length (describing the differences between the edge region and the bulk of the sample)

$$
\ell_{R}=\frac{\ell_{0}}{\sqrt{1+\tilde{\mu}^{2} B^{2}}},
$$

with $\tilde{\mu}<\mu$. This modification turns out [42] to effectively account for the following issues: (i) electron-hole asymmetry, (ii) energy dependence of the electron-hole recombination length and mobility, and (iii) spatial inhomogeneity of the sample. In Figs. 4 and 5 we used $\tilde{\mu} \approx 0.5 \mu$.

Electron-hole asymmetry manifests itself in the nonzero Hall resistivity at charge neutrality. Moreover, for any value of the carrier density, the Hall resistivity is a nonmonotonous function of the magnetic field. As mentioned above, this effect also leads to the apparent drift of the charge neutrality point (in terms of the applied gate voltage) with the external magnetic field.

At temperatures lower than the Debye energy the dominant recombination process involves electrons and holes near the bottom of the band. Indeed, far away from the neutrality point, kinematic constraints preclude the "direct" process where an electron from the upper band is scattered into an empty state in the lower band by means of single acoustic phonon emission. Instead, such "hot" electrons require an additional scatterer (e.g., an impurity [43] or a second phonon) for recombination to take place. In contrast, electrons close to the neutrality point in bilayer graphene are slow enough so that direct, singlephonon recombination is allowed. Hence, within the kinetic equation approach [42], the effective length scale describing the recombination processes depends on energy. Similarly, the impurity scattering time or carrier mobility is, strictly speaking, energy dependent as well. Now, the macroscopic description of Ref. [29] involves quantities that are averaged over the quasiparticle spectrum. Taking into account the existence of several distinct recombination processes, we arrive at the conclusion that after thermal averaging, the typical recombination length $\ell_{R}$ may be described by slightly different effective parameters (in particular, the electronic mobility, $\mu \rightarrow \tilde{\mu}$ ) as compared to, e.g., Drude conductivity.

The width dependence of the carrier mobility indicates that the edge region of the sample is characterized by stronger
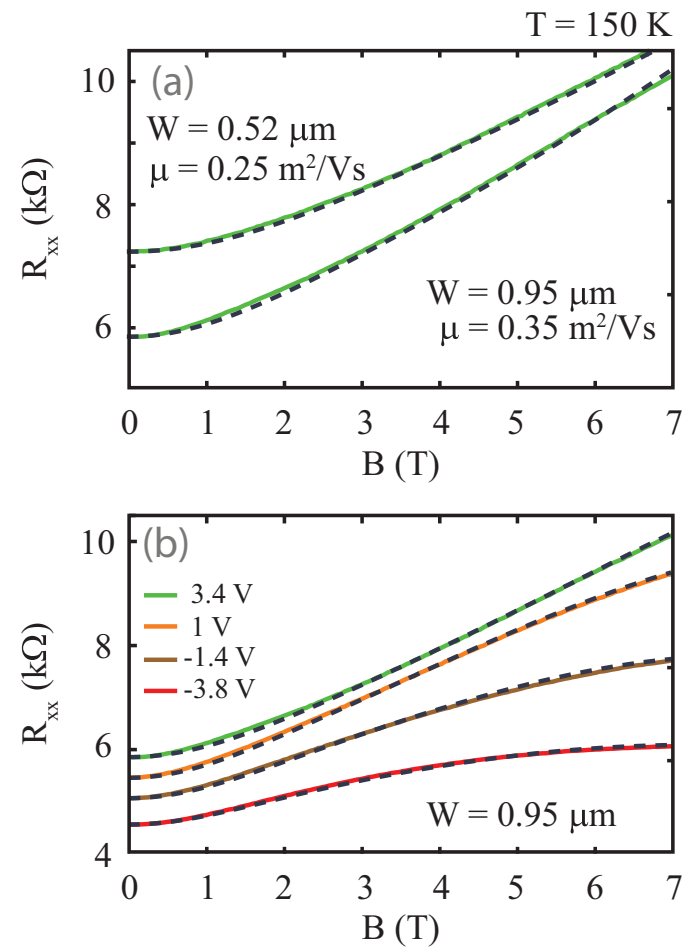

FIG. 5. (a) Magnetoresistance of the narrow (top curves) and medium (bottom curves) sections of the sample at $150 \mathrm{~K}$ and the gate voltage closest to the charge neutrality point $\left(V_{g}=8.2 \mathrm{~V}\right.$ and $V_{g}=3.4 \mathrm{~V}$, respectively). The solid (green) lines represent the experimental data; the dashed (blue) lines represent the theoretical fit using the semiclassical description adapted from Ref. [29] to our sample geometry (see Table I for the complete set of parameters). (b) Magnetoresistance of the medium section of the sample at $150 \mathrm{~K}$ for several values of the gate voltage showing the onset of saturation as the system is tuned away from charge neutrality.

scattering. As a result, all parameters describing electronic transport acquire an effective coordinate dependence across the sample. Since in strong magnetic fields the current is mostly flowing near the sample edges [29], we expect that the effective recombination length $\ell_{R}$ is determined by the lower mobility typical of the near-edge region.

Theoretical results shown in Figs. 4 and 5 were obtained by using expressions (1) with the parameters listed in Table I. The theory (1) assumes that electrons and holes have the same mobility. While plotting Figs. 4 and 5 we have treated the mobility as a free parameter instead of using the values quoted in Sec. II. The reason for this approach is that the experimental estimates of the mobility rely on the Drude-like models of

TABLE I. Microscopic parameters obtained from analyzing the experimental data with the theory (1) for the three sections of the sample (see Figs. 4 and 5).

\begin{tabular}{lccc}
\hline \hline & Narrow & Medium & Wide \\
\hline$W$ & $0.52 \mu \mathrm{m}$ & $0.95 \mu \mathrm{m}$ & $2 \mu \mathrm{m}$ \\
$\mu$ & $0.25 \mathrm{~m}^{2} / \mathrm{V} \mathrm{s}$ & $0.35 \mathrm{~m}^{2} / \mathrm{V} \mathrm{s}$ & $0.42 \mathrm{~m}^{2} / \mathrm{V} \mathrm{s}$ \\
$\ell_{0}$ & $0.43 \mu \mathrm{m}$ & $0.79 \mu \mathrm{m}$ & $1.2 \mu \mathrm{m}$ \\
\hline \hline
\end{tabular}


electronic transport that do not take into account interaction effects, most notably the mutual friction of electrons and holes. Far away from the CNP and in the disorder-dominated regime such models describe the observed effects more or less accurately. Here, the mutual friction is ineffective since with exponential accuracy only one band is partially filled and contributes to low-energy physics. In contrast, close to charge neutrality, the draglike friction effects should be take into account. At the same time, the resulting values of the mobility listed in Table I do not deviate from the values quoted in Sec. II by more than $20 \%$, in accordance with theoretical expectations [44-46].

The values of the recombination length $\ell_{0}$ shown in Table I show a significant dependence on the sample width (roughly, $\left.\ell_{0} \sim W\right)$. We interpret this observation as an indication of a much larger recombination length that would characterize a very large (in theory, infinite) sample (if it would be possible to fabricate without strong structural disorder [4,34]). Assuming that the electron-hole recombination is dominated by an electron-phonon interaction (either impurity or edge assisted), we argue that in narrow samples the phonon spectrum is modified (compared to an idealized infinite system), leading to a much shorter recombination length of the order of the sample width.

The Hall resistance (1b) is expected to vanish at the neutrality point. However, as we have already mentioned, in our sample the neutrality point shifts toward higher gate voltages when a strong magnetic field is applied. Recalculating the fielddependent carrier density from the observed dependence of the resistance maximum (see Figs. 1 and 2), we find a reasonable agreement with the results of the theory (1b) and the measured data [see the brown curve in Fig. 4(b)]. As a consistency check of the theory, we have used the fact that the ratio of the Hall resistance (1b) to the longitudinal resistance (1a) is proportional to the charge density $R_{x y} / R_{x x}=\mu B n / \rho$. Using this ratio to extract the field-dependent quantity $\mu B n / \rho$, we then substituted the result into Eq. (1b) and found a "perfect" agreement with the data [see the blue curve in Fig. 4(b)]. At the same time, the longitudinal resistivity (1a) is much less sensitive to small deviations of the charge density. Indeed, the field dependence of the density in our sample does not lead to visible changes in the calculated curve shown in Fig. 4(a).

The shift of CNP with magnetic field was observed directly (see Figs. 1 and 2). Assuming that the maximum of the longitudinal resistivity corresponds to CNP, we can extract the field dependence of the chemical potential (and, hence, carrier densities) from the data. Using the thus obtained dependence, we recalculated the Hall resistance [see the brown curve in Fig. 4(b)]. The result shows reasonable agreement with the data, with the visible deviations perhaps stemming from the mismatch of temperatures in the two data sets in Figs. 2 and 4 ( $T=1.5 \mathrm{~K}$ and $T=150 \mathrm{~K}$, respectively).

Finally, away from the neutrality point, the data show a tendency towards saturation in high magnetic fields [see Fig. 5(b)]. The theoretical fits where performed with a set of parameters depending on the gate voltage and taking into account the shift of CNP with magnetic field. In particular, the mobility appeared to show a slight increase from $0.35 \mathrm{~m}^{2} / \mathrm{V} \mathrm{s}$ (close to CNP; see Table I) to $0.44 \mathrm{~m}^{2} / \mathrm{V} \mathrm{s}$ at $V_{g}=-3.8 \mathrm{~V}$. At the same time, in that range of gate voltages, the recombination length $\ell_{0}$ appears to be almost unchanged from the value shown in Table I.

\section{CONCLUSIONS}

In this paper we reported the experimental observation of linear magnetoresistance in narrow bilayer graphene samples. The observed behavior is in good qualitative agreement with the two-fluid model of Ref. [29]. The observed effect is specific to the charge neutrality point. Away from neutrality the magnetoresistance shows an approximate linear behavior only in a limited intermediate range of magnetic fields, followed by a tendency to saturation. Our observations are incompatible with the quantum theory of Refs. [30,31] and with the random resistor network model of Ref. [28], but are accounted for in the semiclassical theory of two-component compensated systems of Ref. [29].

Using an empirical modification of the simplest theoretical model (1), we were able to describe our data in a quantitative fashion. A microscopic theory accounting for the physics that is beyond the simplest version of the two-fluid model of Ref. [29] should be based on the quantum kinetic equation [42,47]. Further aspects of the phenomenon of linear magnetoresistance will be the subject of future experimental work, especially in novel materials with charge-compensated two-component systems.

\section{ACKNOWLEDGMENTS}

We are grateful to U. Briskot, M. Dyakonov, A. D. Mirlin, M. Schütt, and S. Wiedmann for helpful discussions. This work was supported by the German Research Foundation DFG SPP 1459, the Dutch Science Foundation NWO/FOM 13PR3118, GIF, the EU Network Grant No. FP7-PEOPLE-2013-IRSES "InterNoM," the Humboldt Foundation, the Russian Foundation of Basic Research (Grant No. 15-02-04496-a), the Dynasty Foundation, the Grant of Russian Ministry of Education and Science (Contract No. 14.Z50.31.0021), and the President Grant for Leading Scientific Schools NSh-1085.2014.2.
[1] A. B. Pippard, Magnetoresistance in Metals (Cambridge University Press, Cambridge, UK, 1989).

[2] A. A. Abrikosov, Fundamentals of the Theory of Metals (NorthHolland, Amsterdam, 1988).

[3] In wide samples of graphene monolayers, a square-root magnetoresistance has been observed in Ref. [48] near the Dirac point, resulting from a combination of the linear dispersion of carriers and the short-range character of the scatterers [49].
[4] F. Kisslinger, C. Ott, C. Heide, E. Kampert, B. Butz, E. Spiecker, S. Shallcross, and H. B. Weber, Nat. Phys. 11, 650 (2015).

[5] Z.-M. Liao, H.-C. Wu, S. Kumar, G. S. Duesberg, Y.-B. Zhou, G. L. W. Cross, I. V. Shvets, and D.-P. Yu, Adv. Mater. 24, 1862 (2012).

[6] A. L. Friedman, J. L. Tedesco, P. M. Campbell, J. C. Culbertson, E. Aifer, F. K. Perkins, R. L. Myers-Ward, J. K. Hite, J. Charles R. Eddy, G. G. Jernigan et al., Nano Lett. 10, 3962 (2010). 
[7] O. Pavlosiuk, D. Kaczorowski, and P. Wisniewski, Sci. Rep. 5, 9158 (2015).

[8] S. Wiedmann, A. Jost, C. Thienel, C. Brüne, P. Leubner, H. Buhmann, L. W. Molenkamp, J. C. Maan, and U. Zeitler, Phys. Rev. B 91, 205311 (2015).

[9] R. Ockelmann, A. Müller, J. H. Hwang, S. Jafarpisheh, M. Drögeler, B. Beschoten, and C. Stampfer, Phys. Rev. B 92, 085417 (2015).

[10] W. Wang, Y. Du, G. Xu, X. Zhang, E. Liu, Z. Liu, Y. Shi, J. Chen, G. Wu, and X. Zhang, Sci. Rep. 3, 2181 (2013).

[11] G. M. Gusev, E. B. Olshanetsky, Z. D. Kvon, N. N. Mikhailov, and S. A. Dvoretsky, Phys. Rev. B 87, 081311 (2013).

[12] X. Wang, Y. Du, S. Dou, and C. Zhang, Phys. Rev. Lett. 108, 266806 (2012).

[13] C. M. Wang and X. L. Lei, Phys. Rev. B 86, 035442 (2012).

[14] C. Shekhar, S. Ouardi, G. H. Fecher, A. Kumar Nayak, C. Felser, and E. Ikenaga, Appl. Phys. Lett. 100, 252109 (2012).

[15] J. Feng, Y. Pang, D. Wu, Z. Wang, H. Weng, J. Li, X. Dai, Z. Fang, Y. Shi, and L. Lu, Phys. Rev. B 92, 081306 (2015).

[16] M. Novak, S. Sasaki, K. Segawa, and Y. Ando, Phys. Rev. B 91, 041203 (2015).

[17] A. Narayanan, M. D. Watson, S. F. Blake, N. Bruyant, L. Drigo, Y. L. Chen, D. Prabhakaran, B. Yan, C. Felser, T. Kong et al., Phys. Rev. Lett. 114, 117201 (2015).

[18] N. J. Ghimire, Y. Luo, M. Neupane, D. J. Williams, E. D. Bauer, and F. Ronning, J. Phys.: Condens. Matter 27, 152201 (2015).

[19] T. Liang, Q. Gibson, N. Ali Mazhar, Liu Minhao, R. J. Cava, and N. P. Ong, Nat. Mater. 14, 280 (2015).

[20] C. Shekhar, A. K. Nayak, Y. Sun, M. Schmidt, M. Nicklas, I. Leermakers, U. Zeitler, Y. Skourski, J. Wosnitza, Z. Liu et al., Nat. Phys. 11, 645 (2015).

[21] F. Arnold, C. Shekhar, S.-C. Wu, Y. Sun, M. Schmidt, N. Kumar, A.G. Grushin, J.H. Bardarson, R. Donizeth dos Reis, M. Naumann, M. Baenitz, H. Borrmann, M. Nicklas, E. Hassinger, C. Felser, and B. Yan, arXiv:1506.06577v2.

[22] Y. Zhao, H. Liu, J. Yan, W. An, J. Liu, X. Zhang, H. Wang, Y. Liu, H. Jiang, Q. Li et al., Phys. Rev. B 92, 041104 (2015).

[23] J. Hu and T. F. Rosenbaum, Nat. Mater. 7, 697 (2008).

[24] A. Husmann, J. B. Betts, G. S. Boebinger, A. Migliori, T. F. Rosenbaum, and M.-L. Saboungi, Nature (London) 417, 421 (2002).

[25] R. Xu, A. Husmann, T. F. Rosenbaum, M. L. Saboungi, J. E. Enderby, and P. B. Littlewood, Nature (London) 390, 57 (1997).

[26] M. Y. Azbel, Zh. Eksp. Teor. Fiz. 44, 983 (1963) [Sov. Phys. JETP 17, 667 (1963)].

[27] I. M. Lifshitz, M. Y. Azbel, and M. I. Kaganov, Electron Theory of Metals (Consultants Bureau, New York, 1973).

[28] M. M. Parish and P. B. Littlewood, Nature (London) 426, 162 (2003).

[29] P. S. Alekseev, A. P. Dmitriev, I. V. Gornyi, V. Y. Kachorovskii, B. N. Narozhny, M. Schütt, and M. Titov, Phys. Rev. Lett. 114, 156601 (2015).
[30] A. A. Abrikosov, Zh. Eksp. Teor. Fiz. 56, 1391 (1969) [Sov. Phys. JETP 29, 746 (1969)].

[31] A. A. Abrikosov, Phys. Rev. B 58, 2788 (1998).

[32] J. Klier, I. V. Gornyi, and A. D. Mirlin, Phys. Rev. B 92, 205113 (2015).

[33] G. Zala, B. N. Narozhny, and I. L. Aleiner, Phys. Rev. B 65, 020201 (2001).

[34] B. Butz, C. Dolle, F. Niekiel, K. Weber, D. Waldmann, H. B. Weber, B. Meyer, and E. Spiecker, Nature (London) 505, 533 (2014).

[35] M. Veldhorst, M. Snelder, M. Hoek, C. G. Molenaar, D. P. Leusink, A. A. Golubov, H. Hilgenkamp, and A. Brinkman, Phys. Status Solidi 7, 26 (2013).

[36] V. N. Abakumov, V. I. Perel, and I. Yassievich, in Nonradiative Recombination in Semiconductors, Modern Problems in Condensed Matter Sciences Vol. 33 (Elsevier, Amsterdam, 1991).

[37] M. Titov, R. V. Gorbachev, B. N. Narozhny, T. Tudorovskiy, M. Schütt, P. M. Ostrovsky, I. V. Gornyi, A. D. Mirlin, M. I. Katsnelson, K. S. Novoselov et al., Phys. Rev. Lett. 111, 166601 (2013).

[38] Characterizing the boundary region by a certain length scale $\ell_{\text {edge }}$, we conclude that the edge scattering dominates the observed effects if the sample width is comparable to $\ell_{\text {edge }}$. In the opposite case, $W \gg \ell_{\text {edge }}$, the edge scattering can be neglected. A detailed experimental study of the edge scattering will be presented elsewhere [39].

[39] D. Smirnov, G. Y. Vasileva, J. C. Rode, C. Belke, Y. B. Vasilyev, Y. L.Ivanov, and R. J. Haug, arXiv:1605.03465v1.

[40] J.-M. Poumirol, W. Escoffier, A. Kumar, B. Raquet, and M. Goiran, Phys. Rev. B 82, 121401 (2010).

[41] Y.-B. Zhou, L. Zhang, D.-P. Yu, and Z.-M. Liao, Appl. Phys. Lett. 104, 153103 (2014).

[42] P. S. Alekseev, A. P. Dmitriev, V. Y. Kachorovskii, I. V. Gornyi, M. Titov, and B. N. Narozhny (unpublished).

[43] J. C. W. Song, M. Y. Reizer, and L. S. Levitov, Phys. Rev. Lett. 109, 106602 (2012).

[44] The mutual friction between electrons and hole is similar to the Coulomb drag effect $[45,46]$. In the disorder-dominated regime, the corresponding relaxation rate is proportional to temperature and is small compared to the inverse mean free time $\tau_{e h}^{-1} \sim \alpha^{2} T \ll \tau^{-1}$. Here, $\alpha$ is the dimensionless parameter describing the strength of Coulomb interaction.

[45] B. N. Narozhny and A. Levchenko, Rev. Mod. Phys. 88, 025003 (2016).

[46] M. Schütt, P. M. Ostrovsky, M. Titov, I. V. Gornyi, B. N. Narozhny, and A. D. Mirlin, Phys. Rev. Lett. 110, 026601 (2013).

[47] B. N. Narozhny, I. V. Gornyi, M. Titov, M. Schütt, and A. D. Mirlin, Phys. Rev. B 91, 035414 (2015).

[48] G. Yu. Vasileva, P. S. Alekseev, Yu. B. Vasilyev, Yu. L. Ivanov, D. Smirnov, H. Schmidt, and R.J. Haug, arXiv:1508.04009.

[49] P. S. Alekseev, A. P. Dmitriev, I. V. Gornyi, and V. Yu. Kachorovskii, Phys. Rev. B 87, 165432 (2013). 